\title{
MECHANISMS AND EFFECTS OF DIETARY RESTRICTION ON CNS AND AFFECTIVE DISORDERS
}

\author{
E. Haritov, M. Garalova, J. Tivcheva, T. Angelov, V. Stamenov \\ Department of Pharmacology and Toxicology, Faculty of Medicine, \\ Medical University - Sofia, Bulgaria
}

\begin{abstract}
Neuropsychiatric disorders, including depression contribute significantly to global disability and possess high social and health burden. Management is dominated by pharmacotherapy and psychotherapy; nevertheless, such treatments prevent or treat less than half of the patients, suggesting that alternative approaches are required. Emerging data suggest that diet may be an adjustable risk factor for psychiatric disorders. Caloric restriction (CR) possesses protective effects in almost all organs including the brain. However, the precise molecular pathways of these effects remain uncertain. In this review, we will discuss the putative neurobiological mechanisms of $C R$ on the brain. The article will address also the molecular basis of the antidepressant effects of $C R$, primarily including ghrelin signaling, CREB neurotropic effects and ketone bodies production. Then we will highlight the probable effect of CR on the neuroinflammation, which emerges as a key pathogenetic factor for the majority of neuropsychiatric disorders. Finally, we discuss the so called caloric restriction mimetics, compounds that reproduce properties of CR. Further research will be required to verify the safety and efficacy of CR before a general approval can be proposed to introduce it and its mimetics in clinical practice for the treatment of neuropsychiatric disorders.
\end{abstract}

Key words: brain, caloric restriction, depression, caloric restriction mimetics, neuroinflammation

Corresponding author: Evgeni Haritov, MD, PhD, Department of Pharmacology and Toxicology, Faculty of Medicine, Medical University, 2 Zdrave Street, 1431 Sofia, Bulgaria, e-mail: evgeniharitov@gmail.com

\section{INTRODUCTION}

$\mathrm{M}$ ental disorders are among the principal causes of disability in the world, accounting for $18 \%$ of years lived with a disability [1]. Due to the high occurrence of ordinary mental disorders, the social and heath load associated with these disorders is significant, with up to $\$ 8$ trillion in lost output ascribed to mental, neurological and substance use disorders [2]. Pharmacotherapy, such as antidepressants and psychotherapy are cornerstones of management; however, they prevent less than half of the disease burden, suggesting that further strategies to prevent and treat psychiatric disorders are required. In fact, recent data suggests that despite a significant increase in the use of psychotropic medications and wider accessibility to psychotherapies, the population burden of affective disorders has not reduced, and may be rising [3].

The new field of nutritional psychiatry provides data for diet quality as an adaptable risk factor for mental disorders. Recent systematic reviews investigating the relationship between diet and general mental disorders have shown healthy dietary patterns to be 
inversely connected with the chance of, or risk for, depression [4]. While there had been a proof on the potential utility of omega- 3 fatty acid and folate preparations in affective disorders during prior decades and number of observational investigations focusing on the ingestion of single nutrients or foods [5], the first studies to explore and create a relationship between general diet quality and the common psychiatric disorders, depression and anxiety, were not available until the last decade [6].

Calorie restriction (CR) is defined as a reduction in energy intake without lowering dietary value. This effortless intervention has shown, in a broad range of laboratory animals, to extend lifetime and decrease the prevalence of several age-related disorders [7]. In humans, CR can diminish markers of oxidative stress and inflammation, and can lower cardiovascular disease risk [8]. Nutritional energy restraint also benefits neurons, as suggested by evidences showing that $C R$ protects neurons against dysfunction in experimental models of epilepcy, stroke and neurodegenerative diseases [9]. At present, the calorie restriction has attracted growing interest due to its clear effects on neuroendocrine system and mood state. Both basic and clinical assays confirmed that dietary restriction triggered intracellular signaling pathways that involve stress reactions and neuronal metabolism [10]. Most of them have been documented as crucial regulators and closely linked with the pathogenesis of depression. However, the issue of whether calorie restriction causes positive or negative efforts on psychiatric disorders remains under discussion. Short time and mild calorie restriction, as well as modest exercise were expected to exhibit antidepressant effects, through activating neuroendocrine hormones to compensate energy deficit.

The objective of the current review is to provide a summary of the field of nutritional psychiatry, including debate of the implicated neurobiological mechanisms that are likely modified by a low-caloric diet, and depiction of the controversial effects of calorie restriction in depression by presenting both experimental data and clinical result, aiming to investigate possible biological mechanisms at the background of these efficacies.

\section{CALORIC RESTRICTION}

Recently, CR has gained significant recognition, as some physicians find these diets straightforward to follow. CR is different from fasting and usually refers to a $20-30 \%$ daily reduction in calorie ingestion. CR is used in clinics by physicians and allows patients to drink water ad libitum while consuming a low-calorie food intake for a week or more. This diet is used for weight management and/or disease treatment. More recently, the effects of $\mathrm{CR}$ have been ivestigated in different model organisms to verify the molecular mechanisms by which dietary interventions may alter lifespan in both human and experimental animals. These studies showed that reduced nutrient intake without falling into undernourishment could increase the mean and the maximum lifetime of rats [11]. According to recent studies, judicious $\mathrm{CR}$ in rats enhances performance on behavioral tests for sensory and motor functions [12]. CR can also raise brain function in adult rats, where it has been related with increased synaptic plasticity and increased assembly of new neurons from neural stem cells [13]. In contrast, new studies discovered that rigorous food deficiency might contribute to dieting-related deficits in cognitive function [14].

CR protects the age-assotiated structural changes as well. The hippocampus and prefrontal cortex are key areas for memory and the deterioration in these regions with age, contributes to cognitive decline [15]. A $25 \%$ reduction in calories initiated at adulthood resulted in the conservation of the grey matter volume in both the hippocampus and the subcortical areas in rhesus macaques [16]. Late beginning $\mathrm{CR}$ in rats also protects the age-assotiated alterations in the dendritic spines thickness. The spine densities of the nutritional restricted rats (20-25-month-old) were equivalent to those of 6-month-old animals indicating that $C R$ has a protective effect on age-assotiated loss of dendritic spines [17]. CR of $30 \%$ in aging rats, conserve white matter integrity, energy production and lasting memory [18]. It also reduces age-related synaptic alterations investigated in skeletal neuromuscular junction of aged (30-month-old) mice. In addition, CR in mice has a protective effect on cerebral blood supply and blood-brain barrier, which stimulates neurovascular function and cognitive functions [19] and also contributes against neurodegenerative disorders, like $A D$. Dietary restriction affects the memory and learning skills in humans as well. In older humans, CR has been shown to slow down dementia [20]. There are data that DR improves memory in healthy elderly (average 70 years) humans that were subjected to a reduction in $35 \%$ calorie intake [21]. A study conducted in healthy subjects confirmed that a $20-30 \%$ dietary restriction for 8 months has beneficial effect on physical activities and improvement of affective disorders such as depression. However, the effect on cognitive functions is still uncertain which may be caused by the differences in dietary protocols, the formation of experimental groups and the evaluation of cognitive performance [22]. 
Current studies of CR in humans now permit the study of changes in the brain and clarify some of the mechanisms related to alterations in cognitive functions. $C R$ has been demonstrated to extend lifespan, preventing many age-associated disorders, and it improves the general health of obese humans [23]. It was also discovered that $\mathrm{CR}$ has a significant role in regulating both cardiovascular and brain functions. Furthermore, CR can efficiently extend lifespan, preventing most age-associated disorders by ameliorating some risk factors for ischemic heart disease, such as increased blood pressure and decreased insulin sensitivity [24]. On the other hand, long-term food limitation was associated with exhaustion, depression, irritability, and reduced heart rate, as well as a variety of self-reported alterations in intellectual functioning, including the failure to concentrate and reduced memory [25]. Dieting to lose weight has also been related to alterations in cognitive function, including poorer performance on a continued attention tasks [26]. This result was replicated in another study that confirmed that in addition to a deficit in constant attention, self-reported dieting was associated with a reduced capacity to remember and slower simple reaction times [25].

Consequently, a combination of judicious CR and exercise could potentially improve brain function and may preclude age-assotiated cognitive decline in humans. It was shown [21] that when the caloric intake of 50 normally fed mature subjects was reduced by $35 \%$ for 6 months, their results on memory tests considerably improved compared with two dissimilar control diet groups [21]. Nourishment can also considerably affect the development of brain functions. Certainly, nutrition provides the appropriate building materials for the brain to form and preserve neural connections, which are decisive for improved cognitive functions [27]. Nutritional factors have extensive and positive actions on neuronal functions [27]. Brain function is indisputably dependent on sufficient nutrition, and short-term modifications in the amount and composition of nutrient intake in humans may affect measures of cognitive function [27].

In summary, diet modification can create positive or negative cognitive outcomes, depending on the dieting model, which is largely influenced by the degree of $\mathrm{CR}$. Current studies on animal models and human subjects showed strong advantageous effects of $C R$ on cognitive function and affective instability [28].

\section{ANTIDEPRESSANT PROPERTIES OF CALORIC RESTRICTION}

Clinicians found that protracted $C R$ reduces negative emotions in patients suffering from eating disorders
[29]. It was shown that fasting and CR noticeably relieved negative moods like nervousness, irritation and bewilderment and enhance the sense of elation among mature men [30]. Moreover, continued CR by $30 \%$ for six months reduced depressive complaints while producing no evident negative effects on humor. In a prospective uncontrolled trial, investigating effects of calorie intake of $300 \mathrm{kcal} / \mathrm{d}$ for 2 weeks in patients suffering from chronic pain was found that, remarkably, more than $70 \%$ of patients showed an efficient improvement in depressive symptoms [31]. It was found that such antidepressant effects were due to augmented availability of neurotransimitters, such as serotonin and norepinephrin. In a different study, 8 days of fasting $(300 \mathrm{kcal} / \mathrm{d})$ induced considerable mood improvement via the polymorphism of GNB3 C825T [32].

Comparable antidepressant effects of $\mathrm{CR}$ were also found in animal models of depression. After CR, mice became more active than their control counter parts [33]. It was also found that CR protected neurons against degeneration in animal models, proposing that CR may protect neurons. In addition, a recent study showed that 14 days of calorie restriction led to a noticeable antidepressant-like effect in rodents [10].

Decreased neurogenesis has been involved in the pathogenesis of anxiety and depression. Influencing hippocampal neurogenesis presents a probable new approach for treating depression. Interestingly, dropping the number of calories consumed promotes the survival of newly created cells in the hippocampus [34]. Moreover, constant mild food restriction activates AMPK following decreased hypothalamic malonyl-CoA, an inhibitor of fatty acid oxidation. Diminished cerebral blood flow is also connected to anxiety and depression. Caloric restriction has been shown to improve cerebral blood flow and blood-brain barrier function in immature mice at 3-4 months of age and is protective for cerebral blood flow in mature rodents. The neurovascular improvements were associated with reduced mammalian target of rapamycin (mTOR) activity, analogous to the effects of the antidepressant ketamine [35].

Acute, short-term and long-term dietetic restrictions have all been shown to stimulate the HPA axis, increasining the levels of glucocorticoids and improving depressive symptoms in a hormetic way [36]. The mechanism by which this increase in glucocorticoids stimulates neuronal endurance and enhances BDNF is not completely known. In rodents, severe caloric restriction rapidly approaches the effects of long-term caloric restriction, suggesting that the favorable effects of caloric restriction may involve only a short-term reduction in caloric intake. In addition, 
the favorable effects induced by a short period of nutritional restriction in adult rodents were maintained even when ad libitum feeding was reintroduced, nevertheless under constant dietary restriction, lifespan extension was more significant in females than in males [37]. On the other hand, in a different study, $80 \%$ of the gene expression effects of continuing caloric restriction were reversed in 8 weeks of return to ad libitum feeding [38].

\section{POSSIBLE MECHANISMS UNDERLYING EFFECTS OF CALORIC RESTRICTION ON THE BRAIN}

A nutrient sensing theory was proposed on how CR improves brain functions and activities. In view of that, during nutrient restriction, an organism is stimulated to use its intelligence in search of food. This causes mild stress on the neurons leading to triggering of signaling pathways that subsequently improve brain activity [28]. The molecular system underlying the positive response of brain function to $\mathrm{CR}$ is a cascade of nutrient-sensing pathways. The key nutrient-sensing pathways are mTOR (mammalian target of rapamycin), AMPK (AMP-activated protein kinase), sirtuins and insulin/ IGF-1, which detect the availability of nutrients and are reviewed briefly below. These pathways control several molecular processes such as metabolism and gene expression [39]. During CR, these diverse nutrient sensors sense the changes in the level of nutrients and the metabolism of the cells, ultimately regulating a range of cellular processes that result in enhanced neuronal plasticity and better cognition [40] (Fig 1).

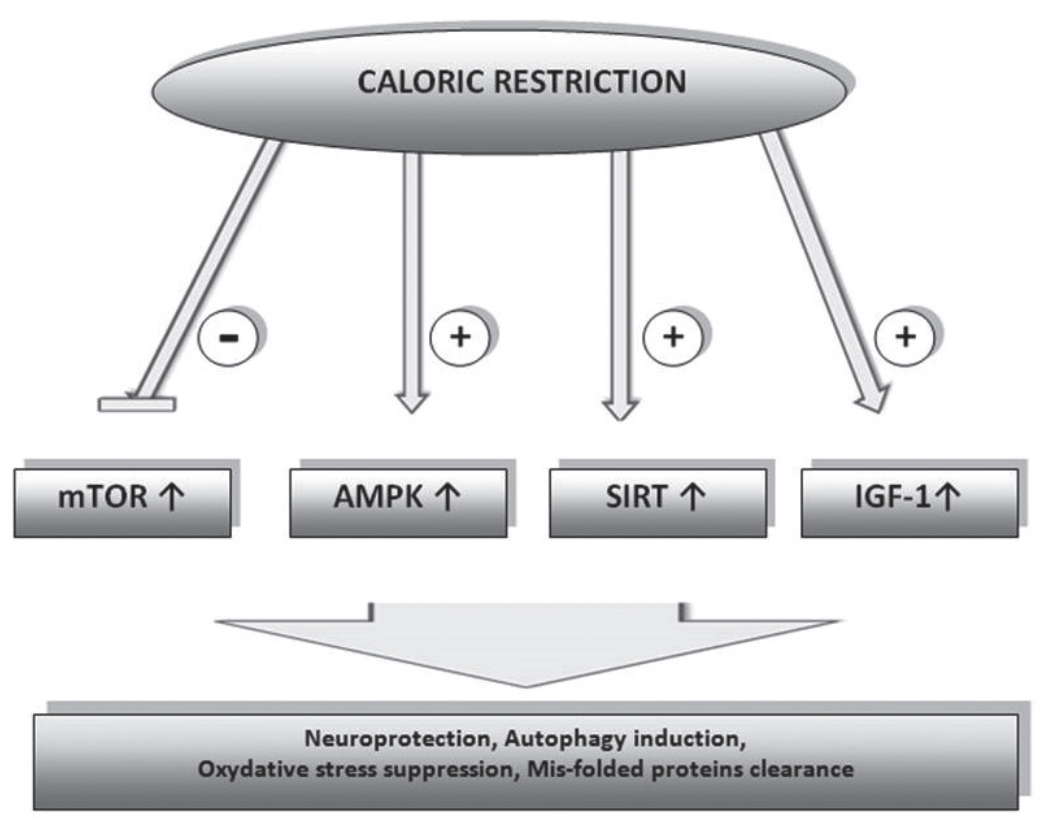

Fig. 1. Molecular mechanisms of neuronal responses to $C R$

\section{Nutrient sensors}

- AMPK. The brain is sensitive to the modifications in the nutrient level, which in turn control feeding and neuroendocrine reactions. Particular neurons are present in the CNS, mainly in the arcuate nucleus in the hypothalamus that senses changes in the levels of glucose [41]. The changes in the level of glucose, detected by hypothalamic neurons, are incorporated into the central nervous system as evident by the augmentation in the level of malonyl-CoA, an inhibitor of beta fatty acid oxidation, through hyperglycemia. When the level of blood glucose is low, such as during caloric restriction, production of malonyl-CoA is suppressed [42]. This is mediated by the activity of AMP-activated protein kinase (AMPK), an evolutionarily preserved serine/threonine kinase, whereby its activation by elevated AMP/ATP status causes the inhibition of Acetyl-CoA carboxylase, an enzyme implicated in the biosynthesis of malonyl-CoA [42].

- mTOR. The limitation of food intake suppresses mTOR (mammalian target of rapamycin) activity in the brain, and is advantageous for the improvement of cognition. When the level of nutrient is inadequate, AMPK is stimulated which in turn inhibits mTOR. Investigations showed that mTOR also influences feeding behaviour and creation of memory in the hippocampus [43]. The inhibition of mTOR, a negative controller of autophagy, by dietary restriction stimulates autophagy, which in turn protects the hippocampal cells from injure and in that way alleviating the age-associated cognitive decline [44].

- Sirtuins. An additional nutrient sensor that plays a role in brain function is sirtuin. Sirt1, the closest homolog of the mold Sir2 has been shown to be implicated in a lot of physiological functions in response to metabolism and is one of the mediators that trigger the beneficial effect of CR [45]. In the brain, Sirt1 is presenteded in varied areas such as the hippocampus and cortex. It is principally expressed in the neurons. Current studies have revealeded that Sirt1 is broadly expressed in the hypothalamus of rodents under wideranging dietary restriction schedules, and influences food intake and physical activities [46]. In addition, the behavioural regulation, Sirt1 is also implicated in neuroprotection.

- IGF-1. IGF-1, a growth factor, is an essential nutrient sensor that is in- 
volved in many cellular processes. As discussed previously, IGF-1 has a significant role during early brain development and cognitive performance. It was reported that $\mathrm{CR}$ in old age suppresseses the signaling pathway of IGF-1 in the brain and this effect may cause higher expression of $\mathrm{FoxO} 3$ transcription factors, which support the oxidative stress resistance and thus protects from neurodegenerative disorders [47]. Current studies in rodents revealed that the diminution of insulin signaling by CR shifts the energy metabolism in the CNS from glucose to ketone bodies metabolism. It furthermore alleviates insulin resistance and through the suppresion of mTOR pathway, it stimulates the clearance of the so called mis-folded protein, like beta amyloid $(A \beta)$ [18]. Therefore, $C R$ retards the development of $A D$ and is valuable for physilogical brain aging.

\section{POSSIBLE MECHANISMS UNDERLYING ANTIDEPRESSANT EFFECTS OF CALORIC RESTRICTION}

\section{Role of Ghrelin}

Ghrelin is a hormone formed by ghrelin cells in the gastrointestinal tract and functions as a neuropeptide in the brain. Ghrelin plays a crucial role in regulating the supply and rate of use of energy [48]. In response of energy shortage, it induces an efficient feeding response by stimulating growth hormone secretagogue receptors (Ghsr, ghrelin receptor) that exists in the brain. Ghrelin stimulates orexin neurons via inducing c-FOS production in orexin neurons. Ghsr polymorphism was observed in a number of depressive patients, and ghrelin application demonstrated beneficial effects on mood in the patients with depression [49]. Increasing ghrelin levels through CR elicited an antidepressant behavior in the rats forced swim test (FST). The antidepressant effects of ghrelin are mainly dependent on direct or indirect activation of orexin neurons in the hypothalamic area. Such activation is indispensable for the antidepressant-like effect of CR. Nevertheless, protracted activation of the orexin neurons may reduce preproorexin mRNA expression in the hypothalamus, impairing the compensatory capacity of orexin neuronal system [48].

\section{Transcritional factor CREB and BDNF}

The omnipresent transcription factor CREB (CAMP responsive element binding) stimulates CAMP-dependent transcriptional responses to a dietary and growth factor stimulus [50]. In metabolic tissues CREB coordinates the fasting response caused by cathecolamines and glucagone [51]. On the other hand in neuronal cells CREB mediates gene expression pathways of neurotrophins leading to neuronal survival. Furthermore, CREB stimulates genes for BDNF in the neuronal cells and activates neural autophagy [52]. It was shown that phosphorylated cAMP responsive-element binding( $\mathrm{p}-\mathrm{CREB})$ was considerably stimulated in calorie restricted rodents [53].

Feeding manners was found to stimulate the production of brain-derived neurotrophic factor (BDNF) and continued neuronal plasticity, playing a crucial role in the neurogenesis [54]. The cerebral glucose decline stimulated by CR also promotes neurogenesis and neurotrophin synthesis. BDNF is a neurotrophin that plays an essential role in the creation of neuronal networks [55]. The BDNF hypothesis of depression postulates that depression principally results from a dysfunction of BDNF, thus its reinstatement can serve as an efficient therapeutic strategy against depression. BDNF also controls the metabolism of serotonin and synaptic plasticity [56]. More comprehensive studies are still needed to clarify the relationship between CR and BDNF function.

\section{Endorphin production}

Endorphins have been recognized to produce feelings of analgesia and euphoria. Studies on the relationship between sports practice and affective disorders have showed that during modest exercise the brain undergoes a stress, which activates the endorphin production [57]. The release of endogenous endorphines and improved depression was found in 7-10 days of fasting, without considerable loss of weight [58]. Therefore, it was proposed that antidepressant efficacy of $\mathrm{CR}$ might result from augmented endorphin to some degree. Nevertheless, more detailed data are still required to verify this point.

\section{Ketone bodies production}

Ketone plays a decisive role in improving mood, alleviating pain, and protecting brain against hypoglycemia [59]. The antidepressant effects of CR might be reliant on the increased production of ketones. It was proposed that the antiseizure properties of ketone bodies might involve its manifold effects on central neuronal system [59]. However, no direct data supporting such effects of ketones in depression has been yet established.

\section{THE ASSOCIATION BETWEEN CALORIC RESTRICTION AND NEUROINFLAMMATION}

A chief characteristic of many neuropsychiatric disorders is systemic, low-grade chronic inflammation in the brain and all over the body. Particularly, these inflammatory signs are comparable to the ones related 
with obesity and metabolic diseases [60], providing a probable suggestion into why $\mathrm{CR}$ exerts anti-inflammatory effects on neuroimmune alterations associated with affective disorders. Neuroinflammation is a host defense mechanism against injurious stimuli and damage in the CNS. Though, chronic inflammation can be detrimental in normal process of aging as well as in pathological situation, like affective disorders. The CNS is composed of mixed cell types, including neurons, microglia, astrocytes. Though two main glial cell types, astrocytes and microglia, are known to be the most important participants in inflammatory responses in the CNS, it is now well known that all neural cells participate to some extent in the neuroinflammatory reactions. Neuroimmune alterations often manifest as microgliosis, and a raise in secreted inflammatory mediators, such as cytokines and chemokines. Emerging data from clinical and basic studies suggests that neuroinflammation is closely related to the pathogenesis of several neuropsychiatric disorders [61].

Although the exact mechanisms of CR's neuroprotective effects are not entirely clarified, it has been suggested that CR exerts its effects through numerous pathways, such as modulating metabolic rates, suppressing oxidative stress, regulating insulin responses, and stimulating neurogenesis [62]. All of the molecular modifications induced by CR may directly or not directly contribute to the regulation of neuroimmune responses associated with neuropsychiatric disorders. CR may directly alleviate activation of glial cells and alter expression of inflammatory cytokines and indirectly control neuroinflammation by reducing inflammatory injuries, such as accumulation of toxic proteins and substances (Fig 2).

A gene profiling study provided data that $C R$ increased $\mathrm{lkBa}$, which is a transcription factor NF-kB inhibitor, and diminished the p65 subunit of NF-kB in rodent neocortex [63]. Besides controling expression of NF-kB, CR also reduced activity of NF-kB in the
CNS of a rat model of experimental astrocytoma [64]. These data suggest that $C R$ suppresses inflammation by inhibiting NF-kB pathways in the CNS. It is well recognized that inflammation enhances ROSgeneration in different cell types. An increase of oxidative stress in the CNS is a feature of aging as well as of some neuropsychiatric disorders. It is obvious that $\mathrm{CR}$ reduces oxidative stress in senescent microglial cells as well as in matured brains, as documened by reduction in ROS and protein oxidation [62]. It is probable that CR suppresses neuroinflammation connected with affective disorders at least partially by reducing oxidative stress and in that way blocking inflammatory responses in the CNS.

Cellular redox condition can also control SIRT1, a regulator of inflammation. SIRT1-mediated deacetylation of p65 subunit of NF-kB suppresses inflammatory reactions by blocking NF-kB signaling pathway [65]. Several lines of evidence have shown that SIRT1 level is increased by CR in different brain areas, such as hippocampus and cortex [66]. It also has been reported that SIRT1 transgenic rats have phenotypes that be similar to CR and show better physical capacity in response to $C R$ than wild-type animals [67]. By contrast, SIRT1-deficient animals demonstrate defects in behavioral responses to $C R$. SIRT1, together with NF-kB, appear to lie at the center of antioxidative and anti-inflammatory responses caused by CR in the CNS.

Apart from NF-kB and SIRT1-mediated cellular cascades, a number of other pathways have been suggested as probable mechanisms mediating the antiinflammatory action of $\mathrm{CR}$, such as modification of BBB permeability and control of steroid hormones in hypothalamic-pituitary-adrenal axis [68]. Nevertheless, it is not clear whether these pathways really mediate anti-inflammatory action of CR in the CNS due to a lack of firm evidence. Additional investigations are necessary to largely understand mechanisms of anti-neuroinflammatory action of CR.

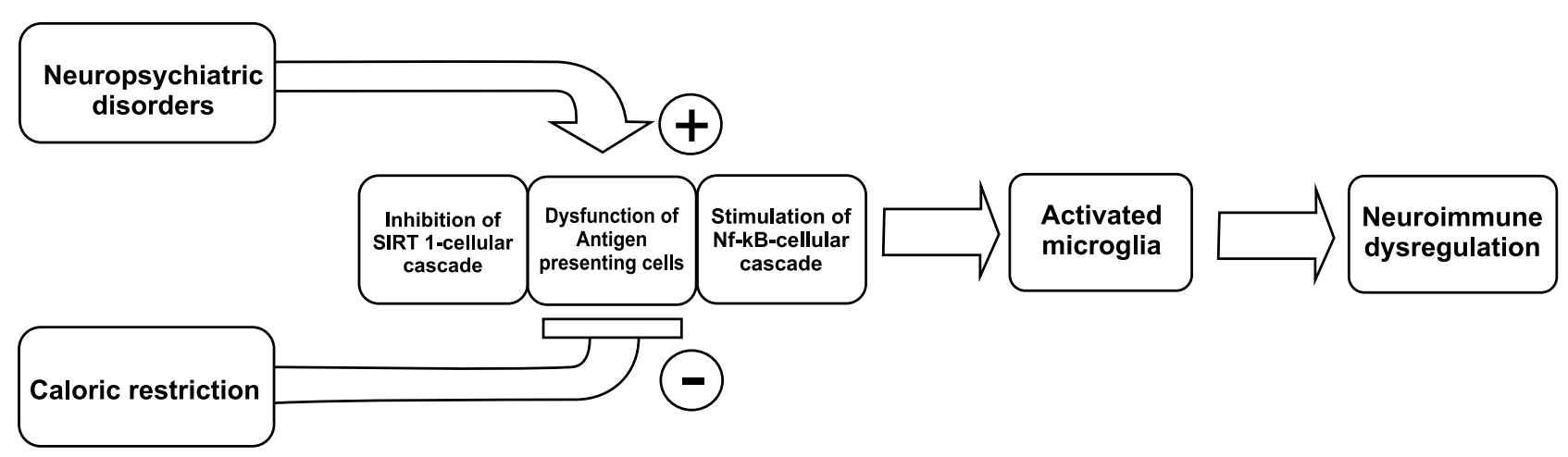

Fig. 2. Putative relationship between neuroimmune alterations in neuropsychiatric disorders and CR 


\section{CALORIC RESTRICTION MIMETICS}

The notion of CR mimetics is quite new. It has been proposed that CR mimetics should have the following key characteristics: 1) they should resemble the metabolic and physiological effects of CR; 2) they should not considerably reduce long-term food intake; 3) they should activate stress-response cellular pathways, as observed in CR, and defend against a range of stressors; and 4) they should decrease inflammation and autoimmune reactions. Given the nutritional concerns of fasting in some patients, CR mimetics, namely pharmacological agents that influence pathways affected by $\mathrm{CR}$, such as rapamycin and metformin, are attractive tools to resemble the defending effects of $\mathrm{CR}$ both for prevention and as adjuvant treatment without nutritional restriction. These CR mimetics influence systemic inflammation and metabolism, and targeting these processes may sensitize neuronal structures in CNS to traditional antidepressants by suppressing neuroinflammation or leading to metabolic modifications in the CNS.

\section{Resveratrol}

Resveratrol is a compound present in high concentration in red grapes. Resveratrol has been demonstrated to prolong the lifespan of nematodes in a sirtuin-dependent mode, which appeared to resemble CR [69]. In addition, variety of studies have shown, that administration of resveratrol could decrease the risk of many age-related disorders [70]. On the other hand, several reports have shown that resveratrol does not posses pro-longevity activity in yeast [70]. Besides, in a study in rats, resveratrol modified the physiology of mature rodents fed with a high-calorie diet towards that of control diet-fed animals, and drastically prolonged their lifespan [71]. Resveratrol causes various changes, as observed in CR rats, including enhanced insulin sensitivity and decreased IGF-I levels. In additional studies, resveratrol was shown to alter gene expression in numerous tissues, with comparable changes to those induced by CR. However, rodents fed with ordinary diet did not live longer when they were administered with resveratrol starting at 12 months of age [72]. These results suggest that the CR-mimetic effects of resveratrol are more obvious in disease conditions, such as obesity and diabetes.

\section{Rapamycin}

Suppression of the target of rapamycin (TOR) signaling cascade by pharmacological application prolongs lifespan in nematodes [73]. In these organisms, CR is mediated, at least partially, by inhibition of TOR signaling. Rapamycin is an antibiotic and immunesuppressor agent. It was shown that rodents ad- ministered with rapamycin to influence the mTOR pathway, have considerably prolonged lifespan in comparison with control animals [74]. These results confirm that the TOR pathway could be a key intracellular signaling cascade that controls a variety of biological mechanisms involved in aging and probably neuropsychiatric disordrs. Consequently, rapamycin and similar molecules that inhibit the TOR pathway may be important candidate $\mathrm{CR}$ mimetics. On the other hand, it is important to underline that there is data showing adverse side-effects of rapamycin such as an increased risk of diabetes [75].

\section{Metformin}

An additional CR mimetic, metformin, is commonly used to treat type 2 diabetes by reducing gluconeogenesis by means of indirect activation of AMP-activated protein kinase (AMPK), thus reducing blood glucose to levels observed in CR rodents [76]. Metformin received a lot of interest after it was verified in a screening assay of drugs demonstrating comparable transcriptional profiles to that of CR in mice [77]. Additionally, metformin was shown to have a CR-related longevity advantage mediated by the stimulation of AMPK in experimental models [78]. Metformin also has a valuable effect on other aspects of the aging course such as a reduction in age-related disease prevalence. These data imply that modulation of AMPK-signaling pathway could be a key target for investigation of $\mathrm{CR}$ mimetics.

\section{CONCLUSION}

Emerging evidences have clarified multiple cellular and molecular levels of neuroprotection by CR. These data are important for our understanding of how metabolism controls brain function and responses to psychiatric disorders and by extension of why neuronal metabolism and neuropsychiatric disorders are tied linked. The antidepressant efficacy of CR in depression has been verified in large number of experimental studies and represents an intriguing therapeutic approach against depression. Scientific data reviewed here suggest that diet could be used to augment the effects of current antidepressants, and reducing their side effects. Clinical studies are continuing and will give confirmation on the possible use of the dietary strategies along with conventional therapeutics.

\section{Acknowledgements \\ This work is supported by the Bulgarian Ministry of Educa- tion and Science under the National Program for Research "Young Scientists and Postdoctoral Students".}




\section{Conflict of interest disclosures}

The authors have no conflict of interest to declare

\section{REFERENCES}

1. Whiteford HA, Ferrari AJ, Degenhardt L et al. The global burden of mental, neurological and substance use disorders: an analysis from the global burden of disease study. PLoS ONE. 2015; 10: e0116820.

2. Chisholm D, Sweeny K, Sheehan $P$ et al. Scaling-up treatment of depression and anxiety: a global return on investment analysis. Lancet Psychiatry. 2016; 3: 415-424.

3. Jorm AF, Patten SB, Brugha TS et al. Is the increased provision of treatment reduced the prevalence of common mental disorders? Review of the evidence from four countries. World Psychiatry. 2017; 16: 90-99.

4. Jacka FN, Cherbuin N, Anstey KJ et al. Does reverse causality explain the relationship between diet and depression? J Affect Disord. 2015; 175: 248-250.

5. Morris MS, Fava M, Jacques PF et al. Depression and folate status in the US population. Psychother Psychosom. 2003; 72 (2): 80-87.

6. Sanchez Villegas A, Delgado RodrÃguez M, Alonso A et al. Association of the mediterranean dietary pattern with the incidence of depression: the seguimiento universidad de navarra/university of navarra follow-up (sun) cohort. Arch Gen Psychiatry. 2009; 66 (10): 1090-1098.

7. Masoro EJ. Subfield history: caloric restriction, slowing aging, and extending life. Sci Aging Knowledge Environ. 2003; 8: RE2.

8. Mattson MP, Wan R. Beneficial effects of intermittent fasting and caloric restriction on the cardiovascular and cerebrovascular systems. J Nutr Biochem. 2005; 16(3):129-137.

9. Bruce-Keller AJ, Umberger G, McFall R et al. Food restriction reduces brain damage and improves behavioral outcome following excitotoxic and metabolic insults. Ann Neurol. 1999; 45(1):8-15.

10. Lutter M, KrishnanV, Russo SJ et al. Orexin signaling mediates the antidepressant-like effect of calorie restriction. $J$ Neurosci. 2008; 28(12): 3071-3075.

11. Taormina G, Mirisola MG. Calorie restriction in mammals and simple model organisms. Biomed Res Int. 2014; 6:1-10.

12. Singh Kalra RR, Fults DW. Preuss award 121 leptomeningeal dissemination cascade in medulloblastoma. Neurosurgery. 2014; 61(Suppl 1):198-9.

13. Lee J, Duan W, Mattson MP. Evidence that brain-derived neurotrophic factor is required for basal neurogenesis and mediates, in part, the enhancement of neurogenesis by dietary restriction in the hippocampus of adult mice. J Neurochem. 2002;82(6): 1367-75.

14. Green MW, Elliman NA, Rogers PJ. Lack of effect of shortterm fasting on cognitive function. J Psychiatr Res. 1995; 29(3): 245-53.

15. West, R.L. An application of prefrontal cortex function theory to cognitive aging. Psychol Bull. 1996; 120: 272-292.

16. Willette, AA, Coe CL, Birdsill AC et al. Interleukin-8 and interleukin-10 brain volume and microstructure, and the influence of calorie restriction in old rhesus macaques. Age (Dordr). 2013; 35: 2215-2227.

17. Moroi-Fetters SE, Mervis RF, London ED et al. Dietary restriction suppresses age-related changes in dendritic spines. Neurobiol Aging. 1989; 10: 317-322.
18. Guo J, Bakshi V, Lin AL. Early shifts of brain metabolism by caloric restriction preserve white matter integrity and long-term memory in aging mice. Front Aging. 2015; 7 : 213-220.

19. Parikh I, Guo J, Chuang KH et al. Caloric restriction preserves memory and reduces anxiety of aging mice with early enhancement of neurovascular functions. Aging (Albany NY). 2016; 8: 2814-2826.

20. Wahl D, Cogger VC, Solon-Biet SM et al. Nutritional strategies to optimise cognitive function in the aging brain. Ageing Res Rev. 2016; 31: 80-92.

21. Witte $A V$, Fobker M, Gellner $R$ et al. Caloric restriction improves memory in elderly humans. Proc Natl Acad Sci U. S. A. 2009 ; 106: 1255-1260.

22. Redman LM, Ravussin E. Caloric restriction in humans: impact on physiological, psychological, and behavioral outcomes. Antioxid Redox Signal. 2011; 14: 228-275.

23. Mattson MP. Challenging oneself intermittently to improve health. Dose Response. 2014; 12(4): 600-18.

24. Mattson MP, Wan R. Beneficial effects of intermittent fasting and caloric restriction on the cardiovascular and cerebrovascular systems. J Nutr Biochem. 2005; 16(3): 129-37.

25. Green MW, Rogers PJ, Elliman NA et al. Impairment of cognitive performance associated with dieting and high levels of dietary restraint. Physiol Behav. 1994; 55(3): 447-52.

26. Rogers PJ, Green MW. Dieting, dietary restraint and cognitive performance. Br J Clin Psychol. 1993 ; 32(Pt 1):113-6.

27. Meeusen R. Exercise, nutrition and the brain. Sports Med. 2014; 44(Suppl 1): 47-56.

28. Mattson MP. Lifelong brain health is a lifelong challenge: From evolutionary principles to empirical evidence. Ageing Res Rev. 2015; 20: 37-45.

29. Moreno-Dominguez S, Rodriguez-Ruiz S, Fernandez-Santaella MC et al. Impact of fasting on food craving, mood and consumption in bulimia nervosa and healthy women participants. Eur Eat Disord Rev. 2012; 20(6): 461-467.

30. Hussin NM, Shahar S, Teng, NI et al. Efficacy of fasting and calorie restriction (FCR) on mood and depression among ageing men. J Nutr Health Aging. 2013; 17(8): 674-680.

31. Michalsen A, Weidenhammer W, Melchart $D$ et al. Short-term therapeutic fasting in the treatment of chronic pain and fatigue syndromes--well-being and side effects with and without mineral supplements. Forsch Komplementarmed Klass Naturheilkd. 2002; 9(4): 221-227.

32. Michalsen A, Frey UH, Merse $S$ et al. Hunger and mood during extended fasting are dependent on the GNB3 C825T polymorphism. Ann Nutr Metab. 2009; 54(3): 184-188.

33. Govic A, Levay EA, Kent $S$ et al. The social behavior of male rats administered an adult-onset calorie restriction regimen. Physiol Behav. 2009; 96(4-5): 581-585.

34. Duan W, Guo Z, Jiang $\mathrm{H}$ et al. Dietary restriction normalizes glucose metabolism and BDNF levels, slows disease progression, and increases survival in huntingtin mutant mice. Proc Natl Acad Sci U S A. 2003; 100: 2911-6.

35. Parikh I, Guo J, Chuang KH et al. Caloric restriction preserves memory and reduces anxiety of aging mice with early enhancement of neurovascular functions. Aging (Albany NY). 2016; 8: 2814-26.

36. Moore MN, Shaw JP, Ferrar Adams DR et al. Anti-oxidative cellular protection effect of fasting-induced autophagy as a mechanism for hormesis. Mar Environ Res. 2015; 107 : 35-44.

37. Hempenstall S, Picchio L, Mitchell SE et al. The impact of acute caloric restriction on the metabolic phenotype in male C57BL/ and DBA/2 mice. Mech Ageing Dev. 2010; 131: 111-8. 
38. Dhahbi JM, Kim HJ, Mote PL et al. Temporal linkage between the phenotypic and genomic responses to caloric restriction. Proc Natl Acad Sci U S A. 2004; 101: 5524-9.

39. Koubova J, Guarente L. How does calorie restriction work? Genes Dev. 2003; 17: 313-321.

40. Fusco S, Pani G. Brain response to calorie restriction. Cell Mol Life Sci. 2013; 70: 3157-3170.

41. Banks WA. Blood-brain barrier and energy balance. Obesity (Silver Spring). 2006; 14, 234-237.

42. Wolfgang MJ, Cha SH, Sidhaye A et al. Regulation of hypothalamic malonyl- CoA by central glucose and leptin. Proc Natl Acad Sci U. S. A. 2007; 104: 19285-19290.

43. Garelick MG, Kennedy BK. TOR on the brain. Exp Gerontol. 2011; 46: 155-163.

44. Dong W, Wang R, Ma LN et al. Influence of age-related learning and memory capacity of mice: different effects of a high and low caloric diet. Aging Clin Exp Res. 2016; 28: 303-311.

45. Fusco S, Pani G. Brain response to calorie restriction. Cell Mol Life Sci. 2013; 70: 3157-3170.

46. Satoh A, Brace CS, Ben-Josef G et al. SIRT1 promotes the central adaptive response to diet restriction through activation of the dorso medial and lateral nuclei of the hypothalamus. $\mathrm{J}$ Neurosci. 2010; 30: 10220-10232.

47. Hadem IKH, Sharma R. Differential regulation of hippocampal IGF-1-associated signaling proteins by dietary restriction in aging mouse. Cell Mol Neurobiol. 2017; 37: 985-993.

48. Harris GC, Wimmer M, Aston-Jones. The role for lateral hypothalamic orexin neurons in reward seeking. Nature. 2005; 437(7058): 556-559.

49. Estabrooke IV, McCarthy MT, Ko E et al. Fos expression in orexin neurons varies with behavioral state. J Neurosci. 2001; 21(5): 1656-1662.

50. Mayr B, Montminy M. Transcriptional regulation by the phosphorylation-dependent factor CREB. Nat Rev Mol Cell Biol. 2001; 2(August (8): 599-609.

51. Altarejos JY, Montminy M. CREB and the CRTC co-activators: sensors forhormonal and metabolic signals. Nat Rev Mol Cell Biol. 2011; 12(March(3): 141-51.

52. Seok S, Fu T, Choi SE et al. Transcriptional regulation ofautophagy by an FXR-CREB axis. Nature 2014; 516(December (7529): 108-11.

53. Fusco S, Ripoli $\mathrm{C}$, Podda MV et al. A role for neuronal CAMP responsiveelement binding (CREB)-1 in brain responses to calorie restriction. Proc Natl Acad Sci U. S. A. 2012; 109(2): 621-626.

54. Castren E, Voikar V, Rantamaki T. Role of neurotrophic factors in depression. Curr Opin Pharmacol. 2007; 7(1): 18-21.

55. Huang EJ, Reichardt LF. Trk receptors: roles in neuronal signal transduction. Annu Rev Biochem. 2003; 72: 609-642.

56. Araya AV, Orellana X, Espinoza J. Evaluation of the effect of caloric restriction on serum BDNF in overweight and obese subjects: preliminary evidences. Endocrine. 2008; 33(3): 300-304.

57. Amorosi M. Correlation between sport and depression. Psychiatr Danub. 2014; 26 Suppl 1: 208-210.

58. Molina PE, Hashiguchi $Y$, Meijerink WJ et al. Modulation of endogenous opiate production: effect of fasting. Biochem. Biophys Res Commun. 1995; 207(1): 312-317.

59. Maalouf M, Rho JM, Mattson MP. The neuroprotective properties of calorie restriction, the ketogenic diet, and ketone bodies. Brain Res Rev. 2009; 59(2): 293-315.

60. Sankowski R, Mader S, Valdes-Ferrer SI. Systemic inflammation and the brain: Novel roles of genetic, molecular, and environmental cues as drivers of neurodegeneration. Front Cell Neurosci. 2015; 9: 28.

61. Di Benedetto S, Muller L, Wenger, E. Contribution of neuroinflammation and immunity to brain aging and the mitigating effects of physical and cognitive interventions. Neurosci Biobehav Rev. 2017; 75: 114-128.

62. Van Cauwenberghe C, Vandendriessche C, Libert C. Caloric restriction: Beneficial effects on brain aging and Alzheimer's disease. Mamm Genome. 2016; 27: 300-319.

63. Lee CK, Weindruch R, Prolla TA. Gene-expression profile of the ageing brain in mice. Nat Genet. 2000; 25: 294-297.

64. Mulrooney TJ, Marsh J, Urits I et al. Influence of caloric restriction on constitutive expression of NF-kappaB in an experimental mouse astrocytoma. PLoS ONE. 2011; 6: e18085.

65. Yeung F, Hoberg JE, Ramsey CS et al. Modulation of NF-kappaB-dependent transcription and cell survival by the SIRT1 deacetylase. EMBO J. 2004; 23: 2369-2380.

66. Spaulding CC, Walford RL, Effros RB. Calorie restriction inhibits the age-related dysregulation of the cytokines TNFalpha and IL-6 in C3B10RF1 mice. Mech Ageing Dev. 1997; 93: 87-94.

67. Satoh A, Brace CS, Ben-Josef G et al. SIRT1 promotes the central adaptive response to diet restriction through activation of the dorsomedial and lateral nuclei of the hypothalamus. J Neurosci. 2010; 30: 10220-10232.

68. Vasconcelos AR, Cabral-Costa JV, Mazucanti CH et al. The Role of Steroid Hormones in the Modulation of Neuroinflammation by Dietary Interventions. Front Endocrinol. (Lausanne) 2016; 7: 9.

69. Wood JG, Rogina B, Lavu S et al. Sirtuin activators mimic caloric restriction and delay ageing in metazoans. Nature. 2004; 430: 686-689.

70. Sinclair DA. Toward a unified theory of caloric restriction and longevity regulation. Mech Ageing Dev. 2005; 126: 987-1002.

71. Bass TM, Weinkove D, Houthoofd $\mathrm{K}$ et al. Effects of resveratrol on lifespan in Drosophila melanogaster and Caenorhabditis elegans. Mech Ageing Dev. 2007; 128: 546-552.

72. Kaeberlein M, McDonagh T, Heltweg B et al. Substrate-specific activation of sirtuins by resveratrol. J Biol Chem. 2005; 280: 17038-17045.

73. Vellai T, Takacs-Vellai K, Zhang $Y$ et al. Genetics: influence of TOR kinase on lifespan in C. elegans. Nature. 2003; 426: 620.

74. Harrison DE, Strong R, Sharp ZD et al. Rapamycin fed late in life extends lifespan in genetically heterogeneous mice. Nature. 2009; 460: 392-395.

75. Blattler SM, Cunningham JT, Verdeguer $F$ et al. Yin Yang 1 deficiency in skeletal muscle protects against rapamycininduced diabetic-like symptoms through activation of insulin/ IGF signaling. Cell Metab. 2012; 15: 505-517.

76. To K, Yamaza H, Komatsu T et al. Down-regulation of AMPactivated protein kinase by calorie restriction in rat liver. Exp Gerontol. 2007; 42: 1063-1071.

77. Dhahbi JM, Mote PL, Fahy GM. Identification of potential caloric restriction mimetics by microarray profiling. Physiol Genomics. 2005; 23: 343-350.

78. Onken B, Driscoll M. Metformin induces a dietary restrictionlike state and the oxidative stress response to extend $\mathrm{C}$. elegans Healthspan via AMPK, LKB1, and SKN-1. PLoS One. 2010; 5: e8758.

Received and accepted 27.07.2019 